\title{
DENSIDAD POBLACIONAL DE Saguinus oedipus (PRIMATES CALLITRICHIDAE) Y DISPONIBILIDAD DE ALIMENTO VEGETAL, COLOSÓ, SUCRE - COLOMBIA
}

\section{POPULATION DENSITY OF Saguinus oedipus (PRIMATES CALLITRICHIDAE) AND VEGETABLES FOOD AVAILABILITY, COLOSÓ, SUCRE - COLOMBIA}

\author{
Jaime De La Ossa V. ${ }^{1}$, Alejandro De La Ossa-Lacayo ${ }^{2}$ \\ ${ }^{1 *}$ Ph.D. Universidad de Sucre, Facultad de Ciencias Agropecuarias, Grupo de Investigación en Biodiversidad Tropical. Sin- \\ celejo, Sucre - Colombia, e-mail: jaimedelaossa@yahoo.com; ${ }^{2}$ Ecólogo. Ciencias Ambientales, Universidad de Sucre. SUE \\ Caribe. Grupo de Investigación en Biodiversidad Tropical. Sincelejo, Sucre - Colombia, e-mail: alejandrodelaossa@yahoo. \\ com. *Autor para correspondencia
}

Rev. U.D.C.A Act. \& Div. Cient. 17(2): 513-520, Julio-Diciembre, 2014

\section{RESUMEN}

El presente estudio analiza para Saguinus oedipus, endémica del Caribe colombiano, la relación existente entre densidad poblacional y disponibilidad de alimento vegetal, en un fragmento de bosque seco tropical del arroyo Colosó, en el departamento de Sucre, Caribe de Colombia. Los resultados de presencia - ausencia muestran que en el fragmento estudiado, están disponibles 55,8\%, de las familias y $44,9 \%$, de las especies vegetales, que son alimentos reportados para la especie. Particularmente, se registraron, como aprovechadas en alimentación, 17 familias y 26 especies de vegetales, que representa un uso del total establecido del $58,6 \%$ familias y $18,8 \%$ especies. Para los dos grupos detectados, según registros históricos, la densidad poblacional encontrada es comparativamente baja y las proporciones entre sexos, macho: hembra (1:2 y 1:1,6), difieren con lo establecido para la especie. Se determina que la oferta de alimento tiene efecto sobre el tamaño poblacional e influye en las proporciones entre sexos.

Palabras clave: Tití cabeza blanca, alimento vegetal, fragmentación boscosa, densidad poblacional, Colombia.

\section{SUMMARY}

The present study analyzes for Saguinus oedipus, endemic species of the Colombian Caribbean, the existing relationship between populational density and vegetable food availability in a tropical dry forest fragment at the Colosó stream, in the department of Sucre, Colombian. In the forest fragment the results of presence absence show that are available $55.8 \%$ families and $44.9 \%$ species in concordance with the reports as vegetables that are food for S. oedipus. Specifically, were registered as vegetable food 17 families and 26 species, what represents a use of $58.6 \%$ families and $18.8 \%$ species from the total established. For the two detected groups, according to historical registrations, the populational density is comparatively low and the proportions between sexes, males: females (1:2 y 1:1.6) varies notably with the established for the species. Be concluded that the habitat quality related to food offer determines the populational size and influences the proportions between sexes.

Key words: Cotton-top tamarin, vegetable food, forest fragmentation, population density, Colombia.

\section{INTRODUCCIÓN}

Los titíes, se alimentan de cinco tipos primarios de alimentos: insectos, frutos, flores, néctar y exudados vegetales (resina, savia y látex) y, eventualmente, presas animales (Garber, 1984, 1991; Sussman \& Kinzey, 1984; Eisenberg, 1989); sin embargo, utilizan estos alimentos con marcadas diferencias en la frecuencia, estrategias de forrajeo y técnicas de consecución (Sussman \& Kinzey, 1984). Los titíes de los géneros Saguinus y Leontopithecus parecen incluir más frutos en su dieta, que Callithrix y Cebuella. Las proporciones de frutos y de néctar floral en la dieta de los titíes, varían entre 40 y $70 \%$ (Garber, 1984; Sussman \& Kinzey, 1984).

S. oedipus, endémico del Caribe colombiano, comúnmente denominado tití cabeza blanca, tistis o cabecita blanca, en 
medio natural, consume frutos de árboles, de bejucos y de epífitas, siendo secundario, en significación, el consumo de insectos y de néctar; mientras que la ingestión de yemas foliares, hojas, tallos y gomas es esporádica (Neyman, 1978; Savage \& Giraldo, 1990). Los exudados son de gran importancia para los titíes; se han observado altos niveles relativos de consumo de gomas en 19 géneros y 28 especies de primates, siendo de gran valor en la alimentación de los callitríchidos (Garber, 1984).

El Proyecto Tití Colombia (http://proyectotiti.com/espanol/ plantas-en-la-dieta-del-mono-titi.htm) señala que $S$. oedipus, se alimenta de 52 familias, que incluyen árboles, arbustos, palmas, epifitas y bejucos, con registro total de 136 especies.

Los pequeños primates, como S. oedipus, presentan un intestino de tamaño reducido y retienen por corto tiempo el alimento después de su ingestión (Savage \& Giraldo, 1990). Esto es evidente en la época de mayor abundancia de frutos, durante la cual, los animales registran un tiempo de tránsito del alimento, a través del tracto digestivo de, aproximadamente dos horas, lo que ocasiona una disminución en la digestibilidad de la fibra; por lo tanto, en función del tiempo, la menor ganancia energética que le ofrecen los alimentos fibrosos podría ser compensada con la riqueza de energía, que aportan los exudados (Oftedal \& Allen, 1996).

La fragmentación del hábitat tiene como sus tres mayores consecuencias: pérdida del hábitat original, reducción del tamaño del hábitat e incremento del aislamiento entre parches (Simberloff \& Abele, 1982; Saunders et al. 1991), además, de propiciar el deterioro nocivo de la diversidad biótica, dentro del hábitat original (Wilcox, 1980; Wilcox \& Murphy, 1985). La pérdida de hábitat y la fragmentación son proce- sos ecológicos que afectan seriamente la estructura de una población, en especial, si posee una distribución restringida, como sucede con las especies endémicas (Estrada \& Coates-Estrada, 1996).

Los fragmentos de bosque actúan como refugios, ya que después de haberse reducido el área de distribución de la especie, la población es obligada a concentrarse en un reducido espacio (Roncancio et al. 2011), generando presiones y aislamiento físico y biótico (Kattan, 1998), siendo la disponibilidad de alimento una de la principales alteraciones (Chapman, 1990).

El presente estudio tuvo como objetivo la identificación de las especies vegetales y las partes consumidas por dos grupos de S. oedipus, que habitan un fragmento boscoso, en el arroyo Colosó, departamento de Sucre, Colombia. El consumo de los vegetales registrados para esta especie fue comparado con información histórica pertinente, al tiempo que se discute la relación existente entre densidad poblacional, fragmentación y oferta de alimento, dentro del área de estudio.

\section{MATERIALES Y MÉTODOS}

Área de estudio: Fragmento boscoso de 33, 15 hectáreas, ubicado en la ribera del arroyo Colosó, departamento de Sucre, Montes de María $\left(9^{\circ} 28^{\prime} 35^{\prime \prime} \mathrm{N}, 79^{\circ} 20^{\prime} 53^{\prime \prime} \mathrm{O}\right.$ y 9 $28^{\circ} 19^{\prime \prime} \mathrm{N}$, $79^{\circ} 21^{\prime} 06^{\prime \prime}$ O) (Figura 1). Ecológicamente, esta zona constituye un relicto de bosque seco tropical (Bs-T) (Holdridge, 1967); con altura máxima de 102msnm, temperatura media anual de $27^{\circ} \mathrm{C}$ y $1200 \mathrm{~mm}$ anuales de precipitación; con dos épocas climáticas al año: una lluviosa, desde mediados de abril hasta finales de noviembre y, otra seca, desde diciembre hasta comienzos de abril (Cuervo et al. 1986).

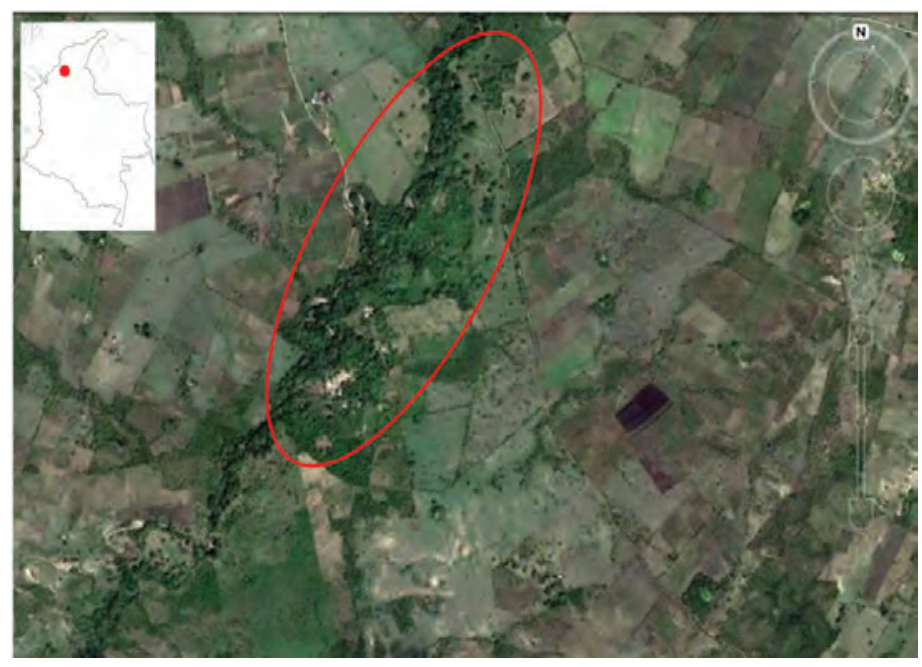

Figura 1. Zona de estudio, arroyo Colosó, Sucre - Colombia (Google Earth, versión libre. https://earth.google.com). 
Colecta de datos: Se seleccionaron al azar 5 parcelas de $10.000 \mathrm{~m}^{2}$ cada una, previa prueba de muestra representativa, con las siguientes condiciones: número máximo de parcelas 33, $\mathrm{p}=0,01$; nivel de confianza alfa $=99 \%$ y error máximo de estimación 10\% (Zar, 1999). En la verificación previa de especies vegetales que hacen parte de la dieta de $S$. oedipus, presentes en el área de estudio, se tuvo en cuenta lo reportado por el Proyecto Tití (http://proyectotiti. com/espanol/plantas-en-la-dieta-del-mono-titi.htm) y se aplicó, en las parcelas establecidas, muestreo de presencia ausencia (Begon et al. 1996). La toma de datos poblacional, se llevó a cabo mediante la aplicación del punto fijo de muestreo (Gallina-Tessaro \& López-González, 2011), usando como puntos, cinco lugares escogidos al azar. Entre marzo y julio de 2011, se trabajó cuatro veces/mes/punto, entre las 06:00 y 12:00 horas.
Análisis de datos: La densidad de población, se calculó como densidad absoluta: número de individuos observados dividido por el área total (Soini, 1992). La identificación del material vegetal colectado, se llevó cabo en la Facultad de Ciencias Agropecuarias de la Universidad de Sucre, de acuerdo con la base de datos de Trópicos. org. (http://www. tropicos.org/home.aspx?langid=66). Los nombres comunes utilizados son de tipo local.

\section{RESULTADOS Y DISCUSIÓN}

En los muestreos de presencia - ausencia de flora, según los registros del Proyecto Tití (http://proyectotiti.com/espanol/plantas-en-la-dieta-del-mono-titi.htm), se identificaron, como alimento disponible en el área de estudio, 29 familias y 62 especies. Como vegetales aprovechados para la alimentación, se detectaron 26 especies, pertenecientes a 17 familias (Tabla 1).

Tabla 1. Especies vegetales disponibles en la zona de estudio, que son alimento de $S$. oedipus, y especies vegetales y partes de éstas, registradas como alimento.

\begin{tabular}{|c|c|c|c|}
\hline \multicolumn{3}{|c|}{ Especies vegetales presentes en la zona de estudio } & \multirow{2}{*}{\begin{tabular}{|l}
$\begin{array}{l}\text { Especies vegetales consumidas } \\
\text { en este estudio }\end{array}$ \\
(F: Fruto, G: Goma, \\
N: Néctar, Fl: Flores
\end{tabular}} \\
\hline Familia & Especie & Nombre común & \\
\hline Acanthaceae & Trichanthera sp. & Palo de agua & \\
\hline \multirow{3}{*}{ Anacardiaceae } & Anacardium excelsum & Caracolí & F, G, Fl. \\
\hline & Astronium graveolens & Santa cruz & \\
\hline & Spondias mombin & Jobo & F, G, Fl. \\
\hline \multirow[t]{2}{*}{ Annonaceae } & Annona purpurea & $\begin{array}{l}\text { Guanábana mat- } \\
\text { imbá }\end{array}$ & \\
\hline & Ephedranthus colombianus & Yaya prieta & $\mathrm{F}$ \\
\hline Apocynaceae & Thevetia ahouai & Tomatillo & \\
\hline \multirow{3}{*}{ Arecaceae } & Philodendron hederaceum & Abrazadera & \\
\hline & Desmoncus orthacanthos & Matamba & \\
\hline & Sabal mauritiiformis & Palma amarga & \\
\hline \multirow{4}{*}{ Bignoniaceae } & Anemopaegma orbiculatum & Bejuco de bacota & \\
\hline & Cydista aequinoctialis & Bejuco colorado & \\
\hline & Pithecoctenium crucigerum & Bejuco canastilla & \\
\hline & Tabebuia rosea & Roble & F, Fl. \\
\hline Boraginaceae & Cordia dentata & Uvito & F, Fl. \\
\hline Cactaceae & Pereskia guamacho & Guamacho & \\
\hline Capparaceae & Capparis baducca & Guanabanito & \\
\hline \multirow{2}{*}{ Celastraceae } & Maytenus longipes & Corocito & $\mathrm{F}$ \\
\hline & Hippocratea volubilis & Corralero & \\
\hline Combretaceae & Combretum fruticosum & Peinecillo & $\mathrm{N}$ \\
\hline Cucurbitaceae & Momordica charantia & Balsamina & \\
\hline
\end{tabular}


Continuación tabla 1




En los muestreos de presencia - ausencia de flora que se registra como alimento para $S$. oedipus, en este estudio, se comprobó que existen, para el área de trabajo, 55,8\% de las familias y 44,9\% de las especies, reportados como vegetales, que son alimento del tití cabeza blanca (http://proyectotiti. com/espanol/plantas-en-la-dieta-del-mono-titi.htm).
En cuanto a la flora, específicamente utilizada por S. oedipus, de acuerdo con lo reportado (http://proyectotiti.com/ espanol/plantas-en-la-dieta-del-mono-titi.htm), se tiene un $58,6 \%$ de familias y un 18,8\% de las especies, que se registran como vegetales, que son alimento para la especie. Al comparar con los resultados de presencia - ausencia del área

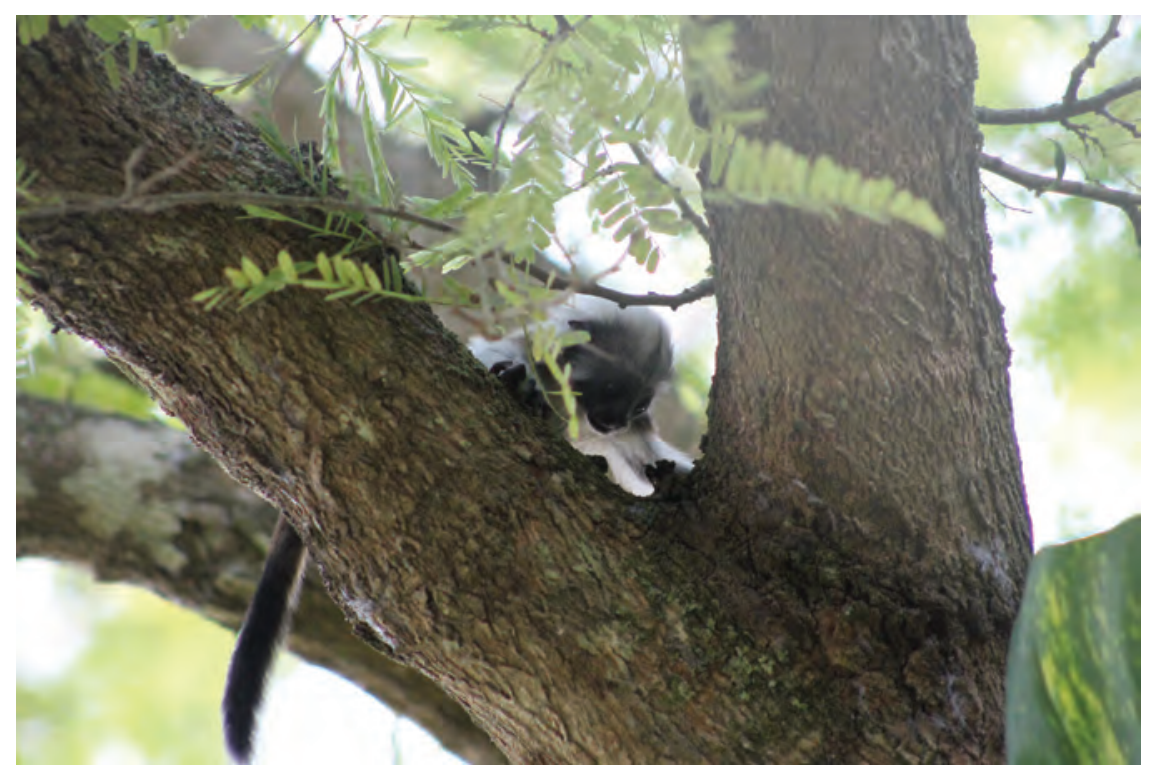

Fotografía 1. Juvenil de Saguinus oedipus, en árbol de tamarindo.

de estudio, se obtuvo una utilización de 62,9\% y de 41,9\% de las familias y especies disponibles, respectivamente.

La utilización del alimento vegetal, se documenta en primates como estacional y variable (Pozo-Montuy \& Serio-Silva, 2006); se conceptúa, además, que se relaciona con el patrón de distribución espacial del bosque (Symington, 1988), con los cambios fenológicos y que influyen en el uso temporal de los sectores dentro de los fragmentos (Milton, 1981; van Schaik et al. 1993). Igualmente, se establece que las condiciones de perturbación del sitio (limitado ámbito hogareño y diversidad florística), generan una alta probabilidad de búsqueda de fuentes alimenticias, que ofrezcan mayor riqueza de nutrientes, ya que se procuraría adquirir mayor volumen de proteína, agua y carbohidratos por unidad de alimento ingerido, aspecto que se relaciona con conductas alimentarias básicas para la sobrevivencia (Serio-Silva et al. 2002), lo que se relacionaría con un forrajeo más intenso sobre algunas áreas y uso preferencial de ciertas especies florísticas.

Los exudados vegetales son una importante fuente de carbohidratos y de minerales, además que se constituyen en alimento de gran importancia en las épocas restrictivas de frutos (Stevenson \& Rylands, 1988) el consumo de gomas, se registra como componente primordial en la dieta de esta especie, al igual que para otros Callitríchidos.

El consumo de flores es un valioso recurso que también esta especie explora (http://proyectotiti.com/espanol/plantasen-la-dieta-del-mono-titi.htm); en este estudio, se detectó, novedosamente, el consumo de flores de caracolí (Anacardium excelsum), de jobo (Spondias mombin), de roble (Tabebuia rosea), de uvito (Cordia dentata), de arizal (Brownea ariza) y de guácimo (Guazuma ulmifolia), lo que indicaría que este recurso es más utilizado, que lo reportado por Neyman (1978) y Savage \& Giraldo (1990). La ocurrencia de un tránsito digestivo corto, dado el reducido tamaño del intestino y una disminución en la efectividad de asimilación de alimentos fibrosos (Savage \& Giraldo, 1990), hace que ítems alimentarios, como las flores, ricas en energía y relativamente bajas en fibra, sean importantes en su dieta, lo cual, también, se relaciona con lo expuesto para el consumo de exudados (Oftedal \& Allen, 1996).

Para el área de estudio, se detectaron dos grupos, con un total de 17 individuos. Los datos por grupo, en cuanto tiene que ver con la densidad y las relaciones entre sexo y madurez, se presentan en la tabla 2. La densidad encontrada 
oscila entre 21,1 y $30,1 \mathrm{ind} / \mathrm{km}^{2}$; se considera baja, al compararla con los registros históricos, que señalan que puede estar entre 30 y $180 \mathrm{ind} / \mathrm{km}^{2}$ (Neyman, 1978). La relación entre machos y hembras, hallada en este estudio, que está entre $1: 1,6$ y $1: 2$, es inversamente diferente del valor $1,8: 1$, conseguido por Neyman (1978). La relación entre maduros e inmaduros de 1:0,16 y 1:0,25, concuerda con lo calculado para la especie por Neyman (1978) y es mayor a lo hallado por Savage et al. (2010), quienes reportan 683 adultos y 90 inmaduros, que equivaldría a 1:0,13.
Se puede establecer que la calidad del hábitat, en cuanto a oferta de alimento, determina el tamaño poblacional y las proporciones entre sexos (Wilcox \& Murphy, 1985; ArroyoRodríguez \& Mandujano, 2003, 2006b). No todos los parches poseen igualdad de condiciones de calidad (Fahrig \& Merriam, 1994; Fleishman et al. 2002; Deng \& Zheng, 2004), ya que la fragmentación afecta la composición y la estructura de la vegetación (Saunders et al. 1991; Benítez-Malvido, 1998; Laurance et al. 1998; Hill \& Curran, 2003; Fahrig, 2003; Arroyo-Rodríguez \& Mandujano, 2006a, 2007), redu-

Tabla 2. Valores poblacionales para S. oedipus, en la zona de estudio.

\begin{tabular}{|c|c|c|c|c|c|c|c|}
\hline Grupo & M & H & I & Total & $\begin{array}{l}\text { Densidad } \\
\left(\mathbf{i n d} / \mathbf{K m}^{2}\right)\end{array}$ & M:H & Ma:I \\
\hline 1 & 2 & 4 & 1 & 7 & 21,1 & $1: 2$ & $1: 0,16$ \\
\hline 2 & 3 & 5 & 2 & 10 & 30,1 & $1: 1,6$ & $1: 0,25$ \\
\hline
\end{tabular}

( $M=$ macho, $\mathrm{H}=$ hembra. $\mathrm{I}=$ inmaduro, $\mathrm{Ma}=$ maduro).

ciendo la cantidad y la calidad de recursos alimenticios para los primates (Tutin, 1999; Estrada \& Coates-Estrada, 2000; Arroyo-Rodríguez \& Mandujano, 2006b). Entonces, aquellas especies que tengan una dieta estrictamente basada en frutos serán más sensibles a alteraciones del hábitat, que aquellas especies capaces de consumir alimentos alternativos; la calidad de los parches puede jugar un papel muy importante en la dinámica poblacional (Fleishman et al. 2002; Deng \& Zheng, 2004; Mbora \& Meikle, 2004).

El área de estudio muestra limitaciones en la oferta de dieta para S. oedipus (http://proyectotiti.com/espanol/plantasen-la-dieta-del-mono-titi.htm), lo que se refleja en una baja densidad poblacional, al compararla con los resultados reportados por Neyman (1978), para la misma zona, cuando la deforestación era mínima y se tenían mejores condiciones de hábitat. Reconociendo los fuertes impactos que puede causar la fragmentación, se puede establecer que la calidad del hábitat, en cuanto a oferta de alimento, determina el tamaño poblacional y las proporciones entre sexos (Wilcox \& Murphy, 1985; Arroyo-Rodríguez \& Mandujano, 2003). Se espera, como consecuencia, que sus nocivos efectos sean más graves, cuando se trata de endemismos que, por su restringida distribución poblacional, puede llevar a la extinción local. No en vano, S. oedipus se encuentra referenciada como en peligro crítico, por la Unión Internacional para la Conservación de la Naturaleza - IUCN (IUCN, 2010).

Conflicto de Intereses: El manuscrito fue preparado y revisado con la participación de todos los autores, quienes declaramos que no existe ningún conflicto de intereses, que ponga en riesgo la validez de los resultados presentados.

\section{BIBLIOGRAFÍA}

1. ARROYO-RODRÍGUEZ, V.; MANDUJANO, S. 2006a. The importance of tropical rain forest fragments to the conservation of plant species diversity in Los Tuxtlas, Mexico. Biodiv. Conserv. 15:4159-4179.

2. ARROYO-RODRÍGUEZ, V.; MANDUJANO, S. 2006b. Forest fragmentation modifies habitat quality for Alouatta palliata. Int. J. Primatol. 27:1079-1096.

3. ARROYO-RODRÍGUEZ, V.; MANDUJANO, S. 2007. Efectos de la fragmentación sobre la composición y la estructura de un bosque tropical lluvioso mexicano. En: Harvey, C.A.; Sáenz, J.C. (eds.): Evaluación y conservación de la biodiversidad en paisajes fragmentados de Mesoamérica. INBio, Costa Rica. p.199-216.

4. ARROYO-RODRÍGUEZ, V.; MANDUJANO S. 2003. Comparación de la estructura vegetal entre fragmentos desocupados y ocupados por Alouatta palliata mexicana en el sureste de México. Neotrop. Primates. 11:168-171.

5. BEGON, M.; HARPER, J.L.; TOWNSEND, C.R. 1996. Ecología: individuos, poblaciones y comunidades.

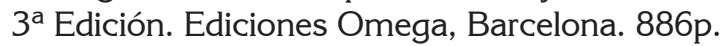

6. BENÍTEZ-MALVIDO, J. 1998. Impact of forest fragmentation on seedling abundance in a tropical rain forest. Conserv. Biol. 2:380-389. 
7. CHAPMAN, C.A. 1990. Ecological constraint opens a group size in three species of neotropical primates. Folia Primatologica. 55:1-9.

8. CUERVO, A.; BARBOSA, C.; DE LA OSSA, V.J. 1986. Aspectos ecológicos y etológicos de primates con énfasis en Alouatta seniculus (CEBIDAE) de la Región de Colosó, Serranía de San Jacinto (Sucre) Costa Norte Colombiana. Caldasia. 14(68-70):709-742.

9. DENG, W.H.; ZHENG, G.M. 2004. Landscape and habitat factors affecting cabot's tragopan Tragopan caboti occurrence in habitat fragments. Biol. Conserv. 111:25-32.

10. EISENBERG, J. 1989. Mammals of the neotropics: The Northwest Neotropics. Vol.1. The University of Chicago. Chicago, Estados Unidos. 449p.

11. ESTRADA, A.; COATES-ESTRADA, R. 1996. Tropical rain forest fragmentation and wild populations of primates at Los Tuxtlas, Mexico. International Journal Primatology. 17 (5):759-781.

12. ESTRADA, A.; COATES-ESTRADA, R. 2000. Contrastes y similitudes en el uso de recursos y patrón diarios de actividades en tropas de monos aulladores (Alouatta palliata) en fragmentos de selva en Los Tuxtlas, México. Neotrop. Primates. 8:131-135.

13. FAHRIG, L. 2003. Effects of habitat fragmentation on biodiversity. An. Rev. Ecol. Evol. Syst. 34:487-515.

14. FAHRIG, L.; MERRIAM, G. 1994. Conservation of fragmented populations. Conserv. Biol. 8:50-59.

15. FLEISHMAN, E.; RAY, C.; SJOGREN-GULVE, P.; BOGGS, C.L.; MURPHY, D.D. 2002. Assessing the roles of patch quality, area, and isolation in predicting metapopulation dynamics. Conserv. Biol. 16:706-716.

16. GALLINA-TESSARO, S.; LÓPEZ-GONZÁLEZ, C. 2011. Manual de Técnicas para el estudio de la Fauna. Universidad Autónoma de Querétaro e Instituto de Ecología, A. C. México. 390p.

17. GARBER, P. 1984. Proposed nutritional importance of plant exudates in the diet of the panamaniam tamarin Saguinus oedipus geoffroyi. International J. Primatology. 5:1-15.

18. GARBER, P. 1991. Seasonal variation in diet and ranging patterns in two species of tamarin monkeys. 1991. Am. J. Phys.l Anthropol. 88:469-482.
19. HILL, J.L.; CURRAN, P.J. 2003. Area, shape and isolation of tropical forest fragments: effects on tree species diversity and implications for conservation. J. Biogeogr. 30:1391-1403.

20. HOLDRIDGE, L.R. 1967. Life Zone Ecology. Tropical Science Center. 1a. ed. San José, Costa Rica. 206p.

21. IUCN. 2010. Saguinus oedipus. Lista Roja de especies amenazadas. Disponible desde Internet en: http:// www.iucn.org/es/ (con acceso 15/11/2012).

22. KATTAN, G. 1998. Transformación de paisajes y fragmentación de hábitat. En: Chávez, M.; Arango, N. (eds): Informe Nacional sobre el estado de la Biodiversidad. Tomo 2. Causas de pérdida de biodiversidad. Instituto Alexander Von Humboldt. Bogotá, Colombia. p.76-82.

23. LAURANCE, W.F.; FERREIRA, L.V.; RANKIN-DE MERONA, J.M.; LAURANCE, S.G.; HUTCHINGS, R.W.; LOVEJOY, T.E. 1998. Effects of forest fragmentation on recruitment patterns in Amazonian tree communities. Conserv. Biol. 12:460-464.

24. MBORA, D.M.N.; MEIKLE, D.B. 2004. Forest fragmentation and the distribution, abundance and conservation of the Tana river red colobus (Procolobus rufomitratus). Biol. Conserv. 118:67-77.

25. MILTON, K. 1981. Food choice and digestive strategies of two sympatric primate species. Am. Nat. 117: 496-505.

26. NEYMAN, P.F. 1978. Aspects of the ecology and the social organitation of free-ranging cotton-top tamarin (Sagunus oedipus) and the conservation status of the species. En: Kleiman, D.G. (ed): Biology and Conservation of the Callitrichidae. Smithsonian Institute Press. Washington, D. C. p.39-71.

27. OFTEDAL, O.T.; ALLEN, M.E. 1996. Wild Mammals in Captivity. En: Kleiman, D.G.; Allen, M.E.; Thompson, K.V.; Lumpkin, S. (eds): University of Chicago Press, Chicago, USA. p.117-128.

28. POZO-MONTUY, G.; SERIO-SILVA. J.C. 2006. Comportamiento alimentario de monos aulladores negros (Alouatta pigra Lawrence, Cebidae) en hábitat fragmentado en Balancán, Tabasco, México. Acta Zoológica Mexicana 22(3): 53-66.

29. PROYECTO TITI. Flora consumida por el tití cabeciblanco. Disponible desde Internet en: http://proyectotiti. 
com/espanol/plantas-en-la-dieta-del-mono-titi.htm (con acceso el 20/03/2012).

30. RONCANCIO, J.N.; ROJAS, W.; DEFLER, T. 2011. Densidad poblacional de Saguinus leucopus en remanentes de bosque con diferentes características físicas y biológicas. Mastozoología Neotropical 18(1):105-117.

31. SAUNDERS, D.A.; HOBBS, R.J.; MARGULES, C.R. 1991. Biological consequences of ecosystem fragmentation: a review. Conserv. Biol. 5:18-32.

32. SAVAGE, A.; THOMAS, L.; LEIGHTY, K.A.; SOTO, L.H.; MEDINA, F.S. 2010. Novel survey method finds dramatic decline of wild cotton-top tamarin population. Nature Communications. p.1-7.

33. SAVAGE, A.; GIRALDO, H. 1990. The ecology of the cotton-top tamarin in Colombia. Am. J. Primatol. 20(2):1-230.

34. SERIO-SILVA, J.C.; RICO-GRAY, V.; HERNÁNDEZ-SALAZAR, V.; ESPINOSA-GÓMEZ, R. 2002. The role of Ficus (Moraceae) in the diet and nutrition of a troop of Mexican howler monkey (Alouatta palliata mexicana) released on an island of southern of Veracruz, Mexico. J. Trop. Ecol. 18(6): 913-928.

35. SIMBERLOFF, D.; ABELE, L.G. 1982. Refuge design and island biogeographic theory: effects of fragmentation. American Naturalist. 120: 41-50.

36. SOINI, P. 1992. Ecología del coto mono (Alouatta seniculus, CEBIDAE) en el río Pacaya, reserva Pacaya - Saimiria, Perú. Folia Amazonica. 4:103-118.

37. STEVENSON, M.F.; RYLANDS, A.B. 1988. The marmosets, genus Callithrix. In: Mittermeier, R.A.; Rylands, A.B.; Coimbra-Filho, A.F.; Fonseca, G.A.B. (Eds).
Ecology and Behavior of Neotropical Primates. Washington, D.C., World Wildlife Fund, p.131-211.

38. SUSSMAN, R.; KINZEY, W. 1984. The ecological role of the Callitrichidae: A Review. Am J Phys Anthropol. 64:419-449.

39. SYMINGTON, M.M. 1988. Food competition and foraging subgroup size in the black spider monkey (Ateles paniscus chamek). Behavior 105: 117-134.

40. TRÓPICOS. ORG. s.f. Nomenclatural, bibliographic, and specimen data. Disponible desde internet en http:// www.tropicos.org/ (con acceso 10/01/2014).

41. TUTIN, C.E.G. 1999. Fragmented living: Behavioural ecology of primates in a forest fragment in the Lopé Reserve, Gabon. Primates. 40:249-265.

42. VAN SCHAIK, C.P.; TERBORGH, J.; WRIGHT, S.J. 1993. The phenology of tropical forest: Adaptative significance and consequences for primary consumers. Ann. Rev. Ecol. Syst. 24: 353-377.

43. WILCOX, B.A. 1980. Insular ecology and conservation. En: Soulé, M.E.; Wilcox, B.A. (eds): Conservation Biology; A evolutionary- ecological perspective. Sinahuer, sunderland-MA. p.95-117.

44. WILCOX, B.A.; MURPHY, D.P. 1985. Conservation strategy: The effects of fragmentationon extinction. Ambiente natural. 125:874-887.

45. ZAR, J.H. 1999. Biostatistical Analysis, $4^{\text {th }}$. Ed. Prentice Hall, Upper Saddle River, NJ. USA. 662p.

Recibido: Febrero 25 de 2014

Aceptado: Agosto 12 de 2014

Como citar:

De La Ossa V., J.; De La Ossa-Lacayo, A. 2014. Densidad poblacional de Saguinus oedipus (Primates Callitrichidae) y disponibilidad de alimento vegetal, Colosó, Sucre, Colombia. Rev. U.D.C.A Act. \& Div. Cient. 17(2): 513-520. 